\title{
Studying nanoscale magnetism and its dynamics with soft X-ray microscopy
}

\author{
Peter Fischer, Member, IEEE
}

\begin{abstract}
Magnetic soft X-ray microscopy allows for imaging magnetic structures at a spatial resolution down to $15 \mathrm{~nm}$ and a time resolution in the sub-100ps regime. Inherent elemental specificity can be used to image the magnetic response of individual components such as layers in multilayered systems. This review highlights current achievements and discusses the future potential of magnetic soft X-ray microscopy at fsec X-ray sources where snapshot images of ultrafast spin dynamics with a spatial resolution below $10 \mathrm{~nm}$ will become feasible.
\end{abstract}

Index Terms-Magnetic soft X-ray microscopy, magnetization reversal, ultrafast spin dynamics, $X$-ray optics.

\section{INTRODUCTION}

$\mathrm{T}$ HE magnetic properties of condensed matter is currently one of most attractive research areas in solid state physics both for fundamental and applied reasons [1].

Research of magnetism in low dimensions has led to important fundamental discoveries, such as the interlayer exchange coupling and the Giant Magnetoresistnace (GMR) effect [2],[3], which is now commonly used e.g. in read head technologies. Solid state magnetism is also a prototype example for the current transition in the way scientific research is performed, i.e. the classical method of observation and interpretation of physical phenomena is extended by the capability and the desire to functionalize and control magnetism down to fundamental length and time scales and also to create novel materials such as multiferroics, which envision revolutionary ways to tailor magnetism.

Recent achievements in state-of-the-art synthesis capabilities, theoretical modeling and the availability of analytical tools have contributed that magnetism has become one of the most important branches in nanosciences with numerous technological applications.

While fundamental length scales, such as magnetic exchange length in magnetism, which are in the sub-10nm regime can be approached to a large extent both experimentally and theoretically with a variety of techniques, the corresponding fundamental time scales down to the fs

Manuscript received October 25, 2007. This work was supported by the Director, Office of Science, Office of Basic Energy Sciences, Materials Sciences and Engineering Division, of the U.S. Department of Energy under Contract No. DE-AC02-05-CH11231.

P. Fischer is with the E.O. Lawrence Berkeley National Laboratory, Center for X-ray Optics, Berkeley, CA 94720 USA, (phone: 510-486-7052; fax: 510486-4550; e-mail: PJFischer@ lbl.gov). regime can be approached by means of optical laser techniques [4], but unfortunately with a limited spatial resolution.

The ultimate question, how magnetism behaves when both the fundamental magnetic length and time scales will be approached cannot be addressed today. A thorough understanding of e.g. the nature and origin of the exchange interaction is therefore still missing.

Magnetic microscopies have largely contributed to the current level of understanding of magnetic phenomena. Spin polarized Scanning tunneling microscopy provides static images with almost atomic spatial resolution [5]. On the other hand time resolved Kerr microscopy using the magneto-optical Kerr effect images with a time resolution down to fs regime, however, as mentioned above with an inherent diffraction limited spatial resolution in the sub-micrometer range only. Furthermore, none of these techniques is able to distinguish the magnetic response from individual components in multicomponent novel materials, which is of paramount interest in the development of novel materials.

The grand challenge is therefore to develop a magnetic microscopy technique that allows for both a sub-10nm spatial resolution with elemental specificity and, at the same time a fsec time resolution with the capability to take instantaneous snapshot images of thus ultrafast spin dynamics.

This review describes the potential of magnetic soft X-ray microcopy, which combines X-ray magnetic circular dichroism as element specific contrast with a high spatial resolution due to Fresnel zone plates used as imaging X-ray optical elements. Using the inherent time structure of current third generation synchrotron sources a sub-100ps time resolution can be achieved and with new fsec X-ray sources which will become available in the near future, the time resolution can be extended into the fsec regime.

\section{EXPERIMENTAL DETAILS}

\section{A. Fresnel zone plate optics}

The fact that the refractive index of soft X-rays is close to unity, has prevented the use of soft X-ray microscopes for almost 80 years after the discovery of X-rays by W.C. Roentgen in 1895.

Fresnel zone plates (FZP), which are now used in X-ray microscope, are circular gratings with a radially increasing line density. These are diffractive elements where the focal length depends on the wavelength of the X-rays. High quality FZP X- 
ray optics can be fabricated by state-of-the-art nanotechnology tools, such as e-beam lithography [6].

FZPs can be designed and therefore customized for specific purposes and applications by a set of parameters, such as

- $\Delta \mathrm{r}$, which is the outermost ring diameter

$-\mathrm{N}$, the number of zones, and

$-\lambda$, the photon wavelength at which the FZP is operating

These three parameters also determine the performance of the FZP in an X-ray microscope, namely

- the spatial resolution is largely given by $\Delta \mathrm{r}$

- the focal length, which is $\sim 4 \mathrm{~N}(\Delta \mathrm{r})^{2} / \lambda$ and

- the spectral bandwidth. $\sim 1 / \mathrm{N}$.

The most advanced FZPs for soft X-ray microscopy have achieved a spatial resolution better than $15 \mathrm{~nm}$ [7], and the current developments seem to make the $10 \mathrm{~nm}$ spatial resolution regime become feasible in the near future. The spectral bandwidth has an impact to the time resolution in soft $\mathrm{X}$-ray microscopy, which is given by the length of the X-ray pulse where the FZP is operating. Current typical FZPs have $\mathrm{N}$ between 500 and several 1000's, therefore the lower fsec time regime is already feasible with regard to the X-ray optics in soft X-ray microscopy.

\section{B. Magnetic full field soft X-ray microscopy}

The optical setup of the full-field soft X-ray microscope endstation XM-1, where the data presented in this review have been obtained is shown in Fig. 1 and described in detail elsewhere [8].

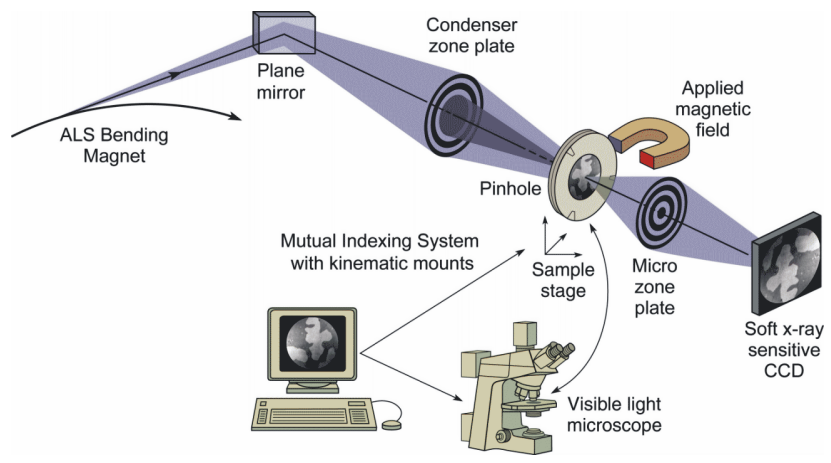

Fig. 1. Optical setup of the full-field high resolution soft X-ray microscope XM-1 at the ALS in Berkeley, CA

Synchrotron radiation emitted from a bending magnet at the Advanced Light Source (ALS) in Berkeley impinges on a condenser zone plate (CZP). The CZP together with a pinhole close to the sample provides a hollow cone illumination of the sample and acts as linear monochromator due its wavelength dependent focal length. With $\mathrm{N} \sim 50,000$ and an outermost zone width of $\sim 40 \mathrm{~nm}$ typical focal lengths of $20-30 \mathrm{~cm}$ can be obtained.. A particular wavelength is chosen by moving the CZP along the optical axis of the synchrotron beam and a spectral resolution of about $1 \mathrm{eV}$ at $500-1000 \mathrm{eV}$ can be achieved. Bending magnet radiation viewed at an angle to the orbital plane of the storage ring provides circular polarization and therefore an aperture upstream the CZP allows to choose circular polarization of different helicity with a typical degree of circular polarization between $60-70 \%$.

$\mathrm{X}$-ray magnetic circular dichroism (XMCD), i.e. the dependence of the photo absorption coefficient on the relative orientation of the projection of the magnetization of the ferromagnetic sample onto the photon propagation direction provides magnetic contrast. In the vicinity of element specific resonant X-ray absorption edges e.g. for $3 \mathrm{~d}$ transition metals such as $\mathrm{Fe}, \mathrm{Co}$, Ni large XMCD values up to $25 \%$ are observed [9].

Illuminating a ferromagnetic sample which exhibits in the groundstate magnetic domains [10], i.e. areas where the magnetization direction varies, with circular polarized X-rays at a photon energy corresponding to absorption edges of a certain ferromagnetic components there is a locally varying transmitted photon intensity.

A second Fresnel zone plate, the microzone plate (MZP), downstream the specimen images the transmitted photons onto a 2dimensional charge coupled device (CCD) detector with a spatial resolution provided by the outermost zone width $\Delta \mathrm{r}$.

The required illumination time is determined by several factors:

- source intensity

- efficiency of the optical elements

- optical thickness of the sample

- quantum efficiency of the detectors.

Since about 1000 photons are required per CCD pixel to yield a sufficient signal-to-noise ratio, the current intensity per $\mathrm{X}$ ray pulse in $3^{\text {rd }}$ generation synchrotron sources is not sufficient to allow for single shot imaging. Typical illumination times are in the second regime and with current X-ray optics a field of view of about $10 \mu \mathrm{m}$ can be achieved.

As a pure photon-in/photon-out based technique magnetic fields of in principle any strength and pointing in any direction can be applied during the recording of X-ray images. At XM-1 typical magnetic fields up to $2-3 \mathrm{kOe}$ in perpendicular geometry and about $1-2 \mathrm{kOe}$ along the plane of the sample can be applied.

Both samples with perpendicular and in-plane anisotropy can be investigated. To image in-plane components the sample has to be tilted at an axis perpendicular to the photon beam propagation [11]. Typically the tilt angle $\alpha$ is $30^{\circ}$, i.e. the effective thickness increases by $1 / \cos \alpha$ thereby reducing the magnetic contrast by a factor 2 .

\section{Time resolved magnetic soft $X$-ray microscopy}

Synchrotron storage rings such as the ALS are inherently pulsed X-ray sources. The electrons having a typical energy of $1.9 \mathrm{GeV}$ circulate in so-called bunches at a velocity close to the speed of light. The typical bunch length corresponds to about $70 \mathrm{ps}$, therefore the emitted X-ray flashes have the same length. This can be used to include time resolution into soft X-ray microscopy and therefore to investigate spin dynamics on the sub-100ps time scale in nanoscale magnetic elements [12]. However, as mentioned above the current available photon intensity per bunch is not sufficient to allow for single shot imaging and therefore a stroboscopic pump-probe scheme has 
to be used. This poses the severe limitation that only perfectly repeatable spin dynamics processes can be studied so far.

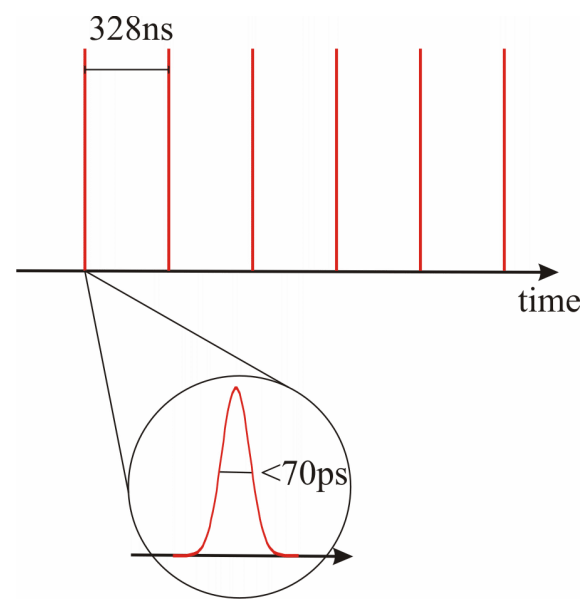

Fig. 2. Time pattern at the ALS during 2.bunch mode operation..The indivdual electron bunches are $328 \mathrm{~ns}$ apart corresponding to $3 \mathrm{MHz}$, while each bunch creates an X-ray pulse of about 70ps length, which determines the time resolution in the stroboscopic mode of operation.

The time resolved studies performed at XM-1 take advantage of the so-called 2-bunch mode operation, where two electron bunches, each $70 \mathrm{ps}$ in width circulate at a $3 \mathrm{MHz}$ frequency, i.e. separated by $328 \mathrm{~ns}$ (Fig. 2)

The scheme for the stroboscopic setup is shown in Fig. 3. The clock signal of the synchrotron triggers a fast electronic pulser, which launches pulses with a rise time of about 100ps into a waveguide structure. These pump pulses can be delayed relative to the X-ray probe pulse. The advent of the X-ray pulse onto the sample is monitored by a fast Avalanche photo diode, which is also displayed on the Oscilloscope.

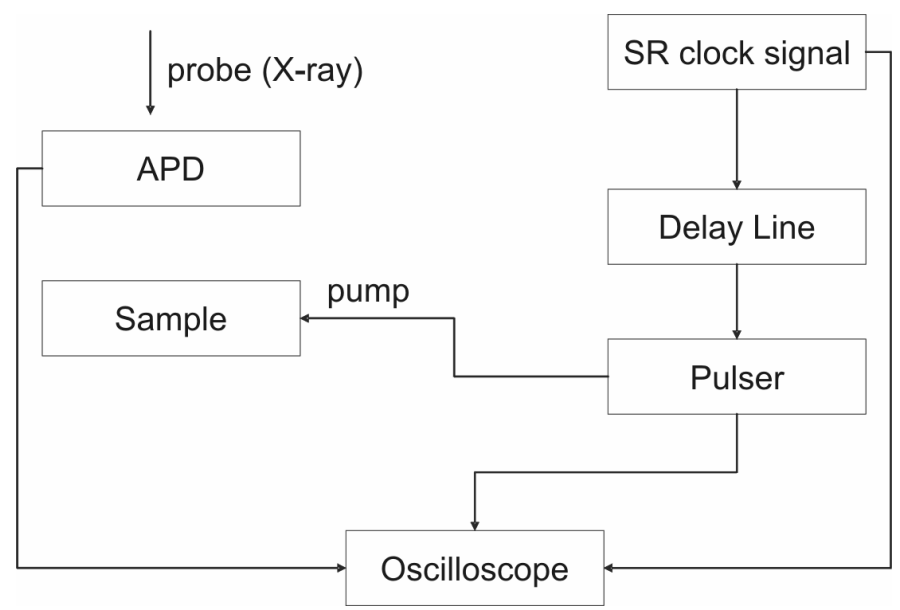

Fig. 3. Box diagram illustrating the pump-probe technique for time resolved soft X-ray microscopy.

Configuring the sample and the waveguide structure, both studies of magnetic field induced spin dynamics as well as spin current induced dynamics can be thus investigated. In the former case the sample is positioned on top of the waveguide
(Fig. 4a) and for the latter the sample is penetrated by the spin current pulse (Fig. 4b) a)

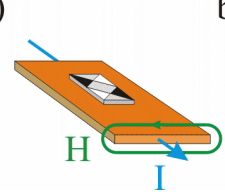

b)

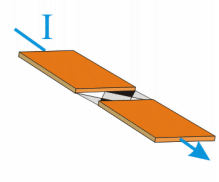

Fig. 4. Schematics of samples on waveguide structure for time resolved soft X-ray microscopy experiment. a) Short pulse creates a Oersted excitation of the element positioned on top of the waveguide. b) The current runs through the sample and creates a spin torque inside the non-collinear magnetic structure.

\section{RESULTS}

\section{A. Studies of magnetization reversal}

The role of interface coupling on magnetization reversal processes in multilayer magnetic thin films is currently being heavily investigated [13], e.g. in the search for suited media for perpendicular recording to enable higher storage density [14]. For example, antiferromagnetically coupled multilayers for materials exhibiting strong perpendicular anisotropy show a surprisingly rich and complex physical behavior [15], [16]. A complete understanding of such systems is still lacking since even 3D micromagnetic calculations are not able to fully describe both interface domain walls at the atomic scale and depict the lateral domain formation at the micrometer scale. Fig. 5 shows results of a magnetic soft X-ray microscopy study in a $[\mathrm{Pt}(0.75 \mathrm{~nm}) / \mathrm{Co}(0.25 \mathrm{~nm})]_{50} / \mathrm{Pt}(3 \mathrm{~nm}) / \mathrm{Tb}_{30} \mathrm{Fe}_{70}$ $(25 \mathrm{~nm}) / \mathrm{Pt}(5 \mathrm{~nm})$ multilayered uncoupled system [17]. The samples were deposited by (co)evaporation onto thin 100nm $\mathrm{Si}_{3} \mathrm{~N}_{4}$ membranes to allow for sufficient penetration of soft $\mathrm{X}-$ rays. Images were recorded at the $\mathrm{Fe}_{3}(706 \mathrm{eV})$ and $\mathrm{Co}_{3}$ $(777 \mathrm{eV})$ absorption edges, i.e. the elemental response from the $\mathrm{Fe}$ and the Co component yields layer resolved image of the magnetic domain structure, which yield a labyrinth pattern with the finest structures in the sub-100nm regime. Interestingly, a comparison of the Fe and the Co containing layers reveals, that the magnetic domains reverse their contrast, which can be easily interpreted as the antiparallel coupling of the two magnetic layers down to the sub-100nm length scale.
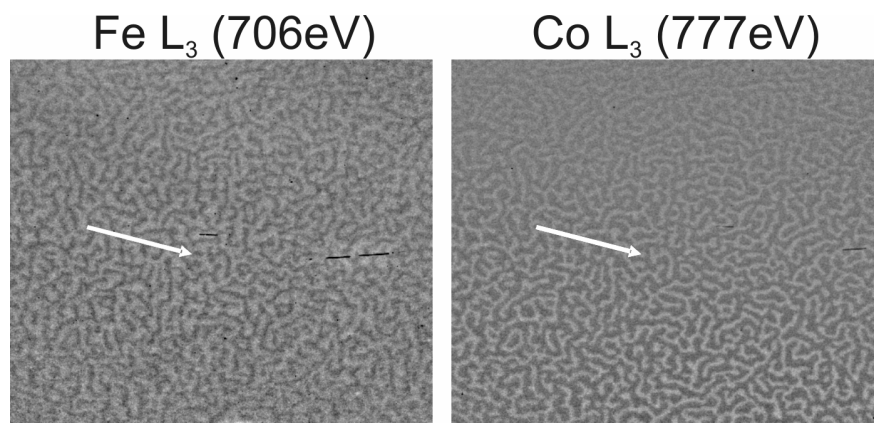

Fig. 5. Layer resolved magnetic domain structure imaged at the $\mathrm{Fe}$ and $\mathrm{Co}$ $\mathrm{L}_{3}$ absortion edges in a $[\mathrm{Pt}(0.75 \mathrm{~nm}) / \mathrm{Co}(0.25 \mathrm{~nm})]_{50} / \mathrm{Pt}(3 \mathrm{~nm}) / \mathrm{Tb}_{30} \mathrm{Fe}_{70}$ $(25 \mathrm{~nm}) / \mathrm{Pt}(5 \mathrm{~nm})$ multilayered film. The antiparallel coupling between the Fe and the Co containing layer is visible by the reversed contrast for any magnetic domain (arrows are use to guide the eye). 
These layer resolved high resolution magnetic soft X-ray microscopy images constitute the first direct imaging of dipolar field induced domain replication in perpendicularly magnetized system.

Another fundamental question associated with the shrinking bit size in high density storage media is whether the domain nucleation during magnetization reversal process exhibits a deterministic behavior [18]. Repeatability of local domain nucleation and deterministic switching behavior are basic and essential factors for achieving high performance in highdensity magnetic recording [19]. High spatial resolution images down to the nanoscale in repeated images of the magnetic domain structure can provide a definite answer. Nanogranular CoCrPt alloy films have received significant attention as a potential high-density perpendicular magnetic recording media. Fig. 6 shows a typical image of the domain configuration of $\left(\mathrm{Co}_{0.83} \mathrm{Cr}_{0.17}\right)_{87} \mathrm{Pt}_{13}$ alloy film recorded at the $\mathrm{Co}_{3}$ absorption edge $(777 \mathrm{eV})$. Since the XMCD signal reflects the local magnetic moment of the Co constituent in this system, integrating the intensity over the field of view shown in Fig. 6a) provides a measure of the macroscopic magnetization for the external magnetic applied during the recording. The $\mathrm{M}(\mathrm{H})$ curve displayed in Fig. 6b) was obtained by recording magnetic transmission soft X-ray microscopy (MTXM) images throughout a full hysteresis scan. The stochastic character can be addressed by analyzing high resolution images taken in repeated $\mathrm{M}(\mathrm{H})$ cycles [20]. The obtained data show a nearly stochastic process in magnetization reversal.

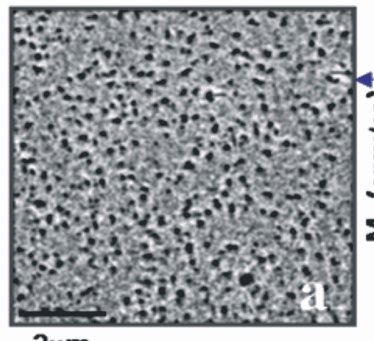

$2 \mu \mathrm{m}$

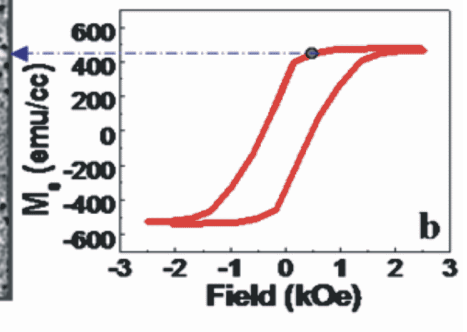

Fig. 6. Typical high resolution $(\Delta \mathrm{r}=15 \mathrm{~nm})$ MTXM image of the nanogranular $\mathrm{CoCrPt}$ layer showing the nucleation sites. The image was recorded at an external field indicated by the position in the $\mathrm{M}(\mathrm{H})$ curve [20].

Instead of reversing magnetization by applying external magnetic (Oersted) fields, the spin torque exerted by a spin polarized current onto a non-linear domain configurations has recently attracted not only significant scientific interest [21], [22], but also as novel pathways for memory [23] and logic [24] devices. The current-driven domain wall motion in magnetic nanowires, particularly the question how fast and how reliable such a domain wall can be moved upon spin current injection is of paramount importance. Numerous experiment measuring e.g. transport properties in such systems have been reported so far [25]. The capability of MTXM to image directly the magnetic domain wall can again contribute significant and novel information.

Curved 60nm thin permalloy wires with a radius of $25 \mu \mathrm{m}$ were prepared by e-beam lithography. Two contact pads were added to the structure to inject $1 \mathrm{~ns}$ short current pulses. To generate a domain wall in between the two contact pads an external magnetic was applied to saturate the magnetization in the wire and subsequently released. In Fig. 7 a) an b) the domain wall before and after injecting a single $1 \mathrm{~ns}$ short pulse with a current density of about $10^{7-8} \mathrm{~A} / \mathrm{cm}^{2}$ is schematically shown. Experimental evidence for the current induced domain wall motion is shown in Fig. 7 c) showing the MTXM image obtained as a difference between the image before and after the injected pulse. This is a direct proof to the current induced domain wall motion. Interesting insight into the speed and the stochastic character of the current induced domain wall motion can be obtained [26]. This is again possible, since a DW moving with a speed of about $110 \mathrm{~m} / \mathrm{s}$ as predicted from micromagnetic simulations travels in 1 nse only $110 \mathrm{~nm}$, therefore high spatial resolution is a prerequisite for such experiments.

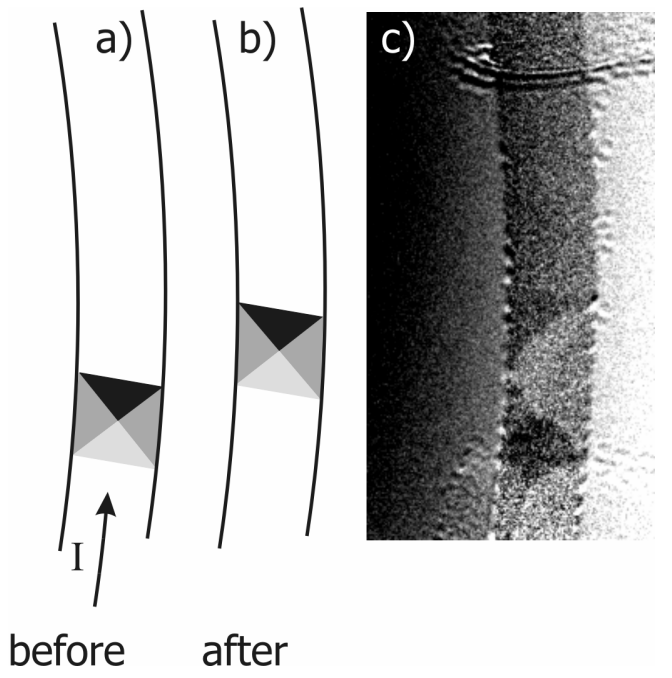

Fig. 7. Schematics of the position of the domain before (a) and after (b) injection of a 1 ns short spin curent pulse into the nanowire. c) Difference MTXM image between a) and b) showing the movement of the domain wall upon spin injection.

\section{B. Imaging sub-ns spin dynamics with high spatial resolution}

The capability to combine the high spatial resolution with soft $\mathrm{X}$-ray microscopy with sub-100ps time resolution is an important step forward to understand spin dynamics on the nanoscale. The groundstate configuration in PY nanoelements are Landau patterns and their perfect repeatability and various features (domain walls, vortices) makes them the ideal candidates for numerous theoretical and experimental studies [27-30].

Spin current induced spin dynamics in such elements is a vastly unexplored area and gives access to an understanding of basic parameters, such as the adiabatic and non-adiabatic contribution to the modified Landau-Lifshitz-Gilbert (LLG) equation of motion incorporating spin torque terms [31]. Rectangular PY elements with a size of $2 \times 4 \mu \mathrm{m}^{2}$ were lithographically prepared and placed in the gap in a waveguide structure (see Fig. 4b). Injecting 1ns short electric pulses through the element generates a precessional motion of the 
domain structure, which is then imaged within the stroboscopic pump-probe setup as described in II.C.

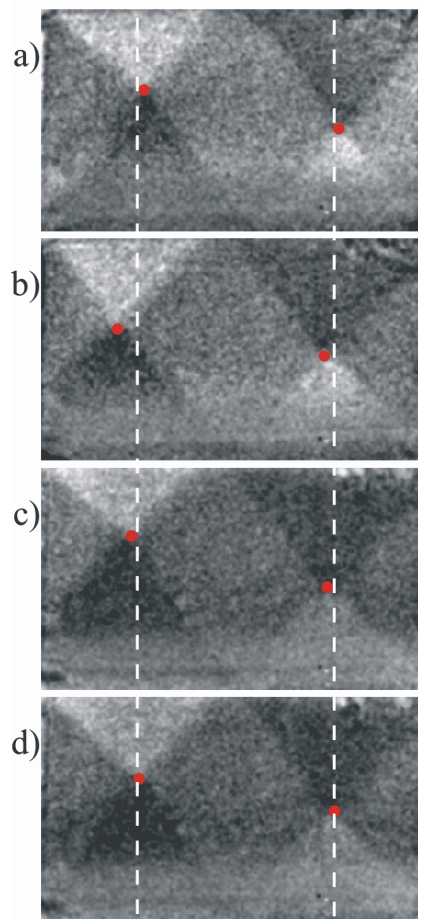

Fig.8. Time resolved MTXM images of a $2 \times 4 \mu \mathrm{m}^{2}$ PY element upon injection of $1 \mathrm{~ns}$ short spin current pulses. The deviation of the vortex position(marked by the red circle) from the dashed line for various delay times between the pump and the probe pulse (a-d) can be clearly seen.

A sequence of images taken at several delay times between 05ns between the pump and the probe pulse are shown in Fig. 8 indicating both the gyrotropic vortex motion as well as the bulging of domain walls. The vertical dashed line serves as a guide to the eye, since the displacement of the vortex amounts to a few 100nm only. The displacement of the vortex in the left part of the image shows a larger displacement than its vortex partner in the right part of the Landau structure. This is due to a enhanced contact resistance in the right part and therefore a reduced current intensity.

\section{CONCLUSION}

Magnetic soft X-ray microscopy has become a valuable tool to study at high spatial resolution magnetization reversal phenomena both induced by classical Oersted as well as by spin current induced phenomena.

Taking advantage of the inherent time structure of synchrotron storage time structure fast spin dynamics with a sub-100ps timescale in magnetic systems can be studied provided it exhibits a perfect repeatability to allow for the application of a stroboscopic pump-probe setup.

Time resolution in soft X-ray microscopy is currently limited by the time structure of the available synchrotron sources. Despite the recent developments to achieve a fsec time resolution even at $3^{\text {rd }}$ generation synchrotron sources employing e.g. slicing techniques [32], [33] or taking advantage of a so-called low- $\alpha$ mode [34], the accompanying severe loss of photon intensity for these mode of operations makes it impossible to use these techniques for imaging at high spatial resolution.

High peak intensity free electron laser (FEL) systems operating in the soft $\mathrm{X}$-ray regime and delivering fsec short $\mathrm{X}$ ray pulses with circular polarization which will become available in the foreseeable future will help to fill this gap and soft X-ray microscopy is a viable instrumentation for that (Fig. 9).

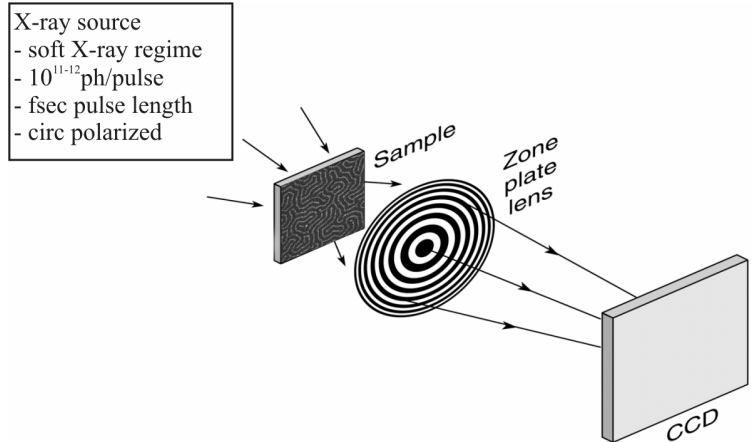

Fig. 9. Schematic setup of full-field soft X-ray microscopy at a fs X-ray source.

Novel experiments, exploring currently completely unknown areas can be tackled by the combination of fsec time and $<10 \mathrm{~nm}$ spatial resolution obtainable within single shot images: non-deterministic processes that appear when the magnetic probe is subject to strong magnetic fields [35], the microscopic picture of all-optical switching [36], the study magnetization far from equilibrium [37], more general the very origin of exchange interaction One can even envision experiments that could completely revolutionize our picture of quantum mechanics, if one has access to experiments beyond the fundamental time scale of exchange interaction.

\section{ACKNOWLEDGMENT}

The author wants to thank D.-H. Kim (now at Chungbuk U, Korea), M-Y. Im, W. Chao, B. Mesler, A. Sakdinawat, B. Gunion, R. Oort, S. Rekawa, D. Kemp, K. Bradley, E. Anderson, D. Attwood, and all the staff from CXRO and ALS. The longstanding and very fruitful collaborations with S.-C. Shin (KAIST, South Korea), with G. Meier, M. Bolte, R. Eiselt, B. Krüger (U Hamburg), and with S. Mangin (U Nancy) are highly appreciated.

\section{REFERENCES}

[1] J. Stoehr and H.C. Siegmann, ,Magnetism”, Springer (2006)

[2] P. Grünberg, R. Schreiber, Y. Pang, M.B. Brodsky and H. Sowers, . Phys. Rev. Lett. 57(19) (1986) pp. 2442-2445

[3] M.N. Baibich, et al. Phys. Rev. Lett. 61(21) (1988) pp. 2472-2475

[4] C.D. Stanciu, F. Hansteen, A.V. Kimel, A. Kirilyuk, A. Tsukamoto, A. Itoh, and Th. Rasing, Phys Rev Lett 99 (2007) pp. 047601-4

[5] M. Bode, Rep. Prog. Phys. 66 (2003) pp. 523-582

[6] D.T. Attwood, "Soft X-rays and Extreme Ultraviolet Radiation: Principles and Applications", Cambridge University Press (1999)

[7] W. Chao, B.H. Harteneck, J.A. Liddle, E.H. Anderson and D.T. Attwood, Nature 435 (2005), pp. 1210-1213

[8] P. Fischer, et al., Rev. Sci. Instr. 72(5) (2001) pp. 2322-24 
[9] C. T. Chen, F. Sette, Y. Ma, and S. Modesti, Phys. Rev. B 42 (1990) pp $7262-7265$

[10] A. Hubert and R. Schäfer, "Magnetic Domains", Springer, Berlin, (1998)

[11] P. Fischer, T. Eimüller, G. Schütz, M. Köhler, G. Bayreuther, G. Denbeaux, and D.T Attwood., J. Appl. Phys., 89 (2001) 7159-7161

[12] P. Fischer, D.-H. Kim, B.L. Mesler, W. Chao, E.H. Anderson, J. Magn. Magn. Mater. 310(2) pt 3 (2007) pp. 2689-2692

[13] J. Nogues and I.K. Schuller, J. Magn. Magn. Mater. 192 (1999) pp. 203232

[14] J.-U. Thiele, S. Maat and E.E. Fullerton, Appl. Phys. Lett. 82, (2003) pp. 2859-2861

[15] O. Hellwig, A. Berger, J. B. Kortright and E.E. Fullerton, J. Magn. Magn. Mater. 319, (2007) pp. 13-55

[16] T. Hauet, J.A. Borchers, Ph. Mangin, Y. Henry, S. Mangin, Phys. Rev. Lett. 96, (2006) pp. 067207-4

[17] S. Mangin, T. Hauet, P. Fischer, D.H. Kim, J.B. Kortright, K. Chesnel, E. Arenholz and Eric E. Fullerton, Phys Rev B (2007) submitted

[18] J. M. Deutsch, A. Dhar, and O. Narayan, Phys. Rev. Lett. $\quad$ (2004), 92, 227203-1

[19] M. Mansuripur, "The Physical Principles of Magneto-optical Recording", Cambridge, University Press, New York (1995), pp. 543676

[20] M.-Y Im, P. Fischer, D.-H. Kim, K.-D. Lee, S.-H. Lee, and S.-C. Shin, Advanced Materials (2007) in print

[21] J. Slonczewski, J. Magn. Magn. Mater. 159, (1996) pp. L1-L7

[22] L. Berger, Phys. Rev. B 54, (1996) pp. 9353-9358

[23] S. S. P. Parkin, US Patent 309,6,834,005 (2004)

[24] D. A. Allwood, Gang Xiong, M. D. Cooke, C. C. Faulkner, D. Atkinson, N. Vernier, R. P. Cowburn., Science 309, (2005). pp. 1688-1692

[25] M Hayashi, L Thomas, C. Rettner, R. Moriya, Y.B. Bazaliy, and S.S.P. Parkin, Phys Rev Lett, 98 (2007) pp. 037204-4

[26] G. Meier, M. Bolte, R. Eiselt, B. Krüger, D.-H. Kim and P. Fischer, Phys Rev Lett., 98 (2007) 187202-4

[27] S.-B. Choe, Y. Acremann, A. Scholl, A. Bauer, A. Doran, J. Stohr, and H.A. Padmore, Science 304, (2004), p 420

[28] K.S. Buchanan, P.E. Roy, M. Grimsditch, F.Y. Fradini, K.Y. Guslienko, S.D. Bader and V. Novosad, Nature Physics 1, (2005) pp. 172-176

[29] J. Raabe, C. Quitmann, C. H. Back, F. Nolting, S. Johnson, and C. Buehler, Phys Rev Lett, 94 (2005) pp. 217204-4

[30] K. Yamada, S. Kasai, Y. Nakatani, K. Kobayashi, Kohno, A. Thiaville and T. Ono, Nature Materials 6, (2007), 269-273

[31] K.-S. Lee and S.-K. Kim. Appl. Phys. Lett. 91 (2007). pp. 132511132514

[32] R.W. Schoenlein, S. Chattopadhyay, H.H.W. Chong, T.E. Glover, P.A. Heimann, C.V. Shank, A.A. Zholents, M.S. Zolotorev, Science 287(5461) (2000) pp.2237-2240

[33] D. Polli, M. Rini, S. Wall, R.W. Schoenlein, Y. Tomioka, Y. Tokura, G. Cerullo, A. Cavalleri, Nature Materials, 6 (2007) pp. 643-647

[34] C. Stamm, et al. Nature Mat. 6 (2007) pp. 740-743

[35] I. Tudosa. et al, Nature 428, 831 (2004)

[36] A.V. Kimel, A. Kirilyuk, P.A. Usachev, R.V. Pisarev A.M. Balbashov and Th. Rasing: Nature 435 (2005) pp. 655-657

[37] L. Perfetti, et al, Phys. Rev. Lett. 99, 197001 (2007). 